\title{
Perturbative discrete-time multivariate fiber channel model with finite memory
}

\author{
Mariia Sorokina, Stylianos Sygletos, and Sergei Turitsyn \\ Aston Institute of Photonic Technologies, Aston University, B4 7ET Birmingham UK \\ m.sorokina@aston.ac.uk
}

\begin{abstract}
We introduce a discrete-time fibre channel model that provides an accurate analytical description of signalsignal and signal-noise interference with memory defined by the interplay of nonlinearity and dispersion. Also the conditional pdf of signal distortion, which captures non-circular complex multivariate symbol interactions, is derived providing the necessary platform for the analysis of channel statistics and capacity estimations in fibre optic links.
\end{abstract}

Keywords: discrete-time channel model, channel memory, nonlinear interference, Shannon capacity

\section{INTRODUCTION}

Accurate description of fibre channel models is critically important for information theory analysis, coding, and digital signal processing techniques in future high capacity transmission systems [1]. Existing channel models [2-5] are based on averaging signal distortions via an infinite memory approximation [6] of the signal interactions, where they are considered to be nonlinear noise. As averaging leads to information loss about the signal interference, it results in a degraded lower bound of the system capacity in the highly nonlinear regime. More accurate channel capacity estimations will require accurate modelling approaches that take into account the finite response of the nonlinear channel [7-8]. These can be also used for other applications such as coding [6] or pre/post-distortion [9-10] compensation, and suggest system designs that can improve significantly the transmission capacity [11-12].

Here we developed a perturbative discrete-time channel model with finite memory based on the nonlinearity compensation technique proposed in [10] and experimentally demonstrated in [11-12]. The resulted multivariate channel model allows us to describe memory effects analytically, including both signal-signal and signal-noise effects. Its accuracy has been compared against numerical simulations and the traditional Gaussian noise model approach, showing high accuracy in every operating regime of the transmission system. Finally, we derive an analytical expression of the conditional pdf that describes signal-interference in fiber-optic channels..

\section{MODEL}

The propagation of a signal via an optical fiber is governed by the nonlinear Schrodinger equation (NLSE):

$$
\frac{\partial U}{\partial z}=-\frac{\alpha}{2} U-i \frac{\beta_{2}}{2} \frac{\partial^{2} U}{\partial z^{2}}+i \gamma|U|^{2} U+\eta(t, z)
$$

where the deterministic distortions are described by the fiber loss parameter $\alpha$, the second-order dispersion parameter $\beta_{2}$, and the nonlinearity coefficient $\gamma$, whereas the random variable $\eta(t, z)$ represents the amplified spontaneous emitted noise by the optical amplifiers along the transmission link.

Given the expansion of the signal over pulses (i.e. $U(t, 0)=\sum x_{k} f(t-k T)$ ), after the matched filter the continuous-time signal $U(t, L)$ undergoes dispersion compensation and sampling. This results in a discrete-time channel model representation with normalized coordinate $\xi=z / L_{d}$ (here $L_{d}$ is dispersion length and $L$ is the transmission length:

$$
Y_{k}^{\prime}=\frac{L_{d}}{L} \eta_{k}+\varepsilon V[Y]_{k}, \quad V[Y]_{k}=\Psi_{s}(\xi) \sum_{m, n=-M}^{M} Y_{k+m}(\xi) Y_{k+n}(\xi) Y_{k+m+n}^{*}(\xi) \tilde{C}_{m n}(\xi), \quad \varepsilon=L_{d} / L_{n l}
$$

where the AWGN noise term $\eta_{k}$ is characterised by the correlation $\left\langle\eta_{k}(\xi), \eta_{k^{\prime}}^{*}\left(\xi^{\prime}\right)\right\rangle=\Psi_{n}(\xi) \delta\left(\xi-\xi^{\prime}\right) \delta_{k k^{\prime}}$ and $\Psi_{n}(\xi)=\left\lfloor\xi L_{d} / L_{s}\right\rfloor^{2} e^{-\alpha \bmod \left(\xi L_{d}, L_{s}\right)} \quad$ is the noise power profile and $\lfloor x\rfloor$ denotes floor function over variable $x$. Also, $\Psi_{s}(\xi)=e^{-\alpha \bmod \left(\xi L_{d}, L_{S}\right)}$ represents the signal power profile and $L_{s}$ is the span length. The coupling 


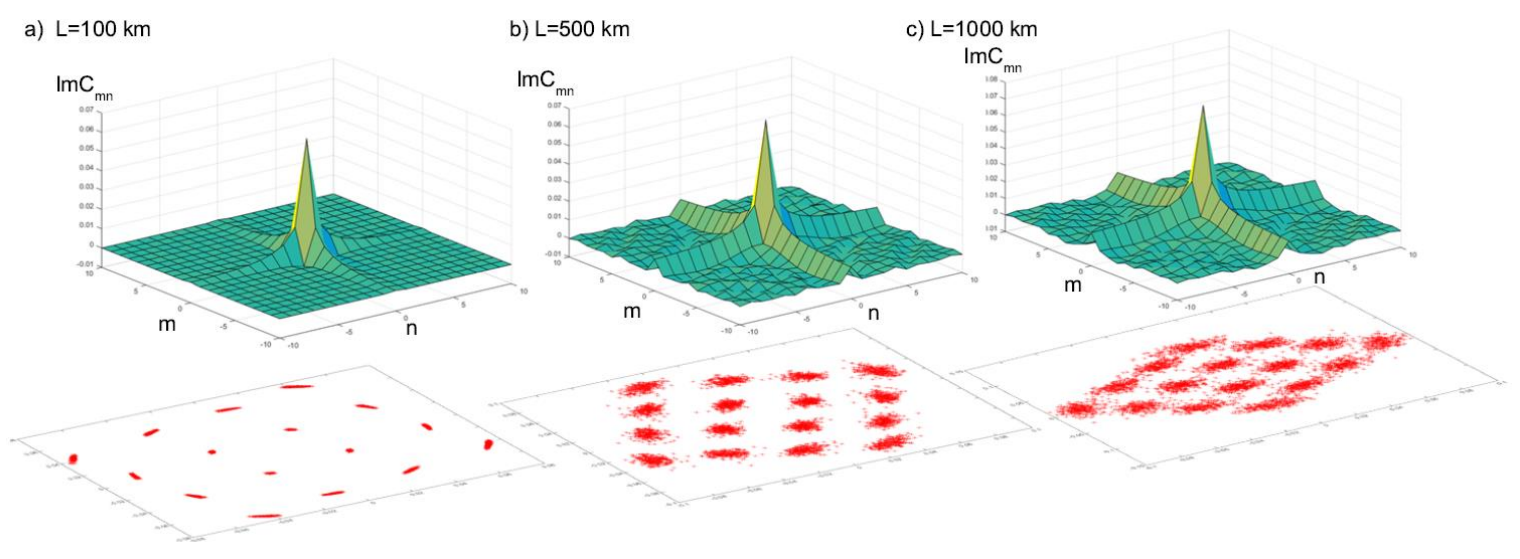

Figure 1. Coupling matrix for various transmission distances a) $L=100 \mathrm{~km}, b) L=500 \mathrm{~km}, \mathrm{c}) \mathrm{L}=1000 \mathrm{~km}$ and the corresponding received constellation diagrams below (for input power $6 \mathrm{dBm}$ ). It is seen that coupling matrix reflects the number and strength of symbol interaction and its effect on signal distortion.

matrix $\tilde{C}_{m n}$ defines the memory behaviour (within a memory window $M$ ) of the transmission channel and depends on its physical properties and the signal pulse shape:

$$
\tilde{C}_{m n}=i \iiint d \omega d \omega^{\prime} d \omega^{\prime \prime} e^{-i \omega^{\prime} \omega^{\prime \prime} \beta_{2} L_{d} \xi-i \omega^{\prime} m T-i \omega^{\prime \prime} n T} f^{*}(\omega) f\left(\omega+\omega^{\prime}\right) f\left(\omega+\omega^{\prime \prime}\right) f^{*}\left(\omega+\omega^{\prime}+\omega^{\prime \prime}\right)
$$

The proposed channel model generalizes a number of previous results. Namely, the class of infinite-memory Gaussian noise models ( $[2,5]$ and references therein) can be received from Eq. 2 after averaging the nonlinear interference term, whereas the finite memory model developed in [6] can be received by averaging of the coupling matrix while keeping the information about interfering symbols.

\section{NUMERICAL VALIDATION}

The coupling matrix governs the signal-signal interactions $C_{m n}=\int d z \Psi_{s}(z) \tilde{C}_{m n}$ which are responsible for the non-circular distribution of the distortion. Also, it describes the impact of different pulse shapes, which is not captured by any of the previous models. Each element of the coupling matrix represents the weight of the interference between symbols in their corresponding time slots. To demonstrate this effect we considered the transmission of a single-channel in a dispersion unmanaged fiber link. For simplicity we used Gaussian pulses of $10 \mathrm{ps}$ full width at half maximum duration and 28GBaud. The link parameters were $\alpha=0.2 \frac{\mathrm{dB}}{\mathrm{km}}, \beta_{2}=$ $-20 \frac{p s^{2}}{k m}, \quad \gamma=1.3 \frac{1}{W k m}, L_{s}=100 \mathrm{~km}$. Figures 1a)-c) reveal that the strength of the interference between symbols decays exponentially with their time difference (denoted by the symbol slot parameters $m$ and $n$ ). At low distances it has a non-uniform shape due to the dominance of the phase distortion (see constellation diagrams below). As the distance increases, the memory increases and as more symbols interact, the resulting distortion at each constellation point becomes more circular, see figures 1b) and 1c). Since the channel matrix can accurately capture the inter-symbol interaction, it can be used as a tool for cancelling the nonlinear distortion at the transceiver/reciever side. This cannot be achieved with the conventional approaches that assume averaging of the signal statistics.

In Figure 2 one can see the achieved agreement between the results of the analytical model (in blue) with those derived from the numerical simulation of the NLSE (in red), as well as, the constellations after the use of the model for pre/post distortion compensation. These have been taken for 16- and 64- QAM signals at power levels of 10 and $12 \mathrm{dBm}$, respectively. In all cases the model is able to provide an accurate description of the signal distortions in the optical fiber. It is also noticed that the distortions cannot be described as circular Gaussian noise, which suggests that such approximation may yield significantly lower estimations of the capacity.

Finally, in Figure 3 we compare achievable data rates (here we used a transmission length of $1000 \mathrm{~km}$ ) for nonlinear distortion uncompensated transmission: the existing GN-based prediction [2,5] is compared with estimations based on calculating the variance of nonlinear distortions given the proposed model. One can see that GN predictions are in accordance with the proposed model and the numerical simulations for large transmission distances. For short distances the deviation from the Gaussian circularity makes the GN approach 

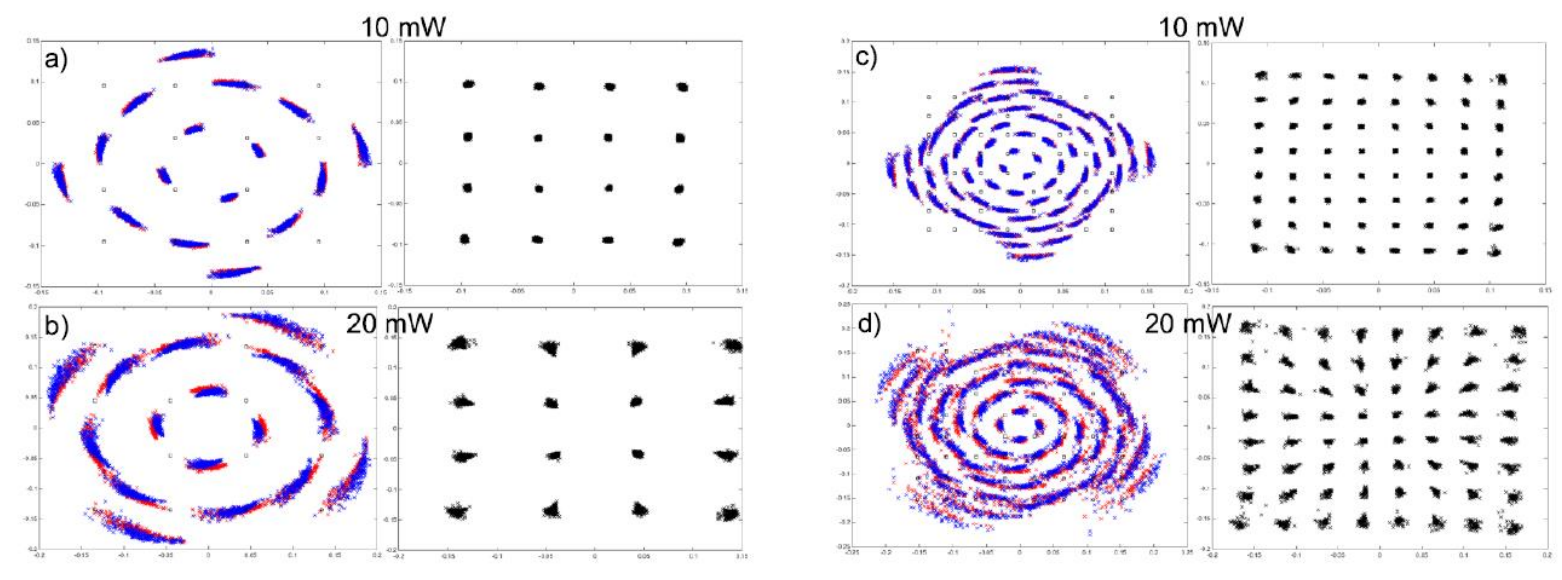

Figure 2. Comparison of numerical (in red) with analytical results (in blue) for 16- (a and b) and 64-QAM (c and d) signals for $10 \mathrm{dBm}$ and $20 \mathrm{dBm}$ input powers. The right side panels show constellations after compensating the transmission distortion. Only deterministic distortions are considered.
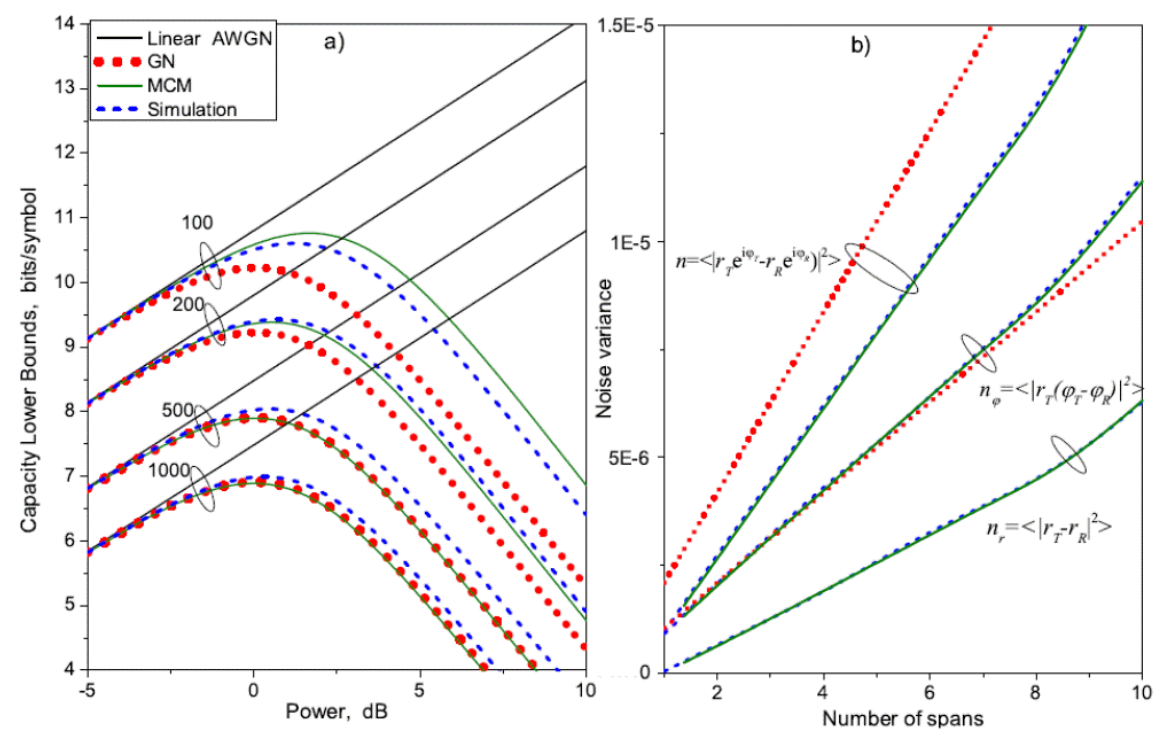

Figure 3. Here we analyze different lower bounds on capacity: a) for uncompensated signal-signal (S-S) distortions, here one can compare estimations based on the existing GN model (red), proposed channel model (green) and numerical simulations (blue). The GN model works well for large transmission distances, however deviates for shorter lengths. This is because GN model does not capture phase and amplitude distributions, which are accurately described by the proposed model as plotted in panel $b$ ).

too pessimistic, whereas our developed model gives accurate description in all cases. This is further illustrated in Figure 3b) where phase, amplitude and total power are plotted. It can be seen that our approach is in a good agreement with the numerical simulations and accuracy describes signal distortions in every power level and transmission distance.

\section{CONDITIONAL PDF AND CAPACITY LOWER BOUNDS}

In the previous estimation of the lower bound Gaussian distribution as input pdf, which is optimum only for linear channels. However, optimizing the input distribution for an accurate conditional pdf, that contains full information about signal interference, can provide more accurate lower bound estimations and, thus, higher transmission rates. To derive tighter bounds one needs to optimize the mutual information functional over input pdf. For that, one must calculate the multivariate conditional pdf that takes into account the memory effects, as it is defined by Eq. 2. Next, we derive this conditional pdf for the transmitted symbols. The channel model represents a mixing of signal and noise components as a result of the inter-symbol interference. A linear combination of univariate independent and identically distributed normal vectors can be represented as a complex normal distribution: 


$$
P(\boldsymbol{Y} \mid \boldsymbol{X})=\pi^{-n}(|\boldsymbol{\Gamma}||\mathbf{P}|)^{-\frac{1}{2}} \exp \left[-\frac{1}{2}\left[(\boldsymbol{y}-\boldsymbol{x})^{H},(\boldsymbol{y}-\boldsymbol{x})^{T}\right]\left(\begin{array}{cc}
\boldsymbol{\Gamma} & \boldsymbol{Y} \\
\boldsymbol{Y} & \boldsymbol{\Gamma}
\end{array}\right)\left(\begin{array}{c}
\boldsymbol{y}-\boldsymbol{x} \\
(\boldsymbol{y}-\boldsymbol{x})^{*}
\end{array}\right)\right]
$$

where * means the complex conjugate, $\mathrm{T}$ means transposition, and $\mathrm{H}$ means transposition and complex conjugate. The covariance matrix $\boldsymbol{\Gamma}$ (complex, non-negative definite and Hermitian) and relation matrix $\mathbf{Y}$ (complex and symmetric) are given as:

$$
\boldsymbol{\Gamma}=\mathrm{E}\left[(\boldsymbol{Y}-\boldsymbol{X})(\boldsymbol{Y}-\boldsymbol{X})^{H}\right], \quad \boldsymbol{Y}=\mathrm{E}\left[(\boldsymbol{Y}-\boldsymbol{X})(\boldsymbol{Y}-\boldsymbol{X})^{T}\right], \quad \boldsymbol{P}=\boldsymbol{\Gamma}^{*}-\boldsymbol{Y}^{H} \boldsymbol{\Gamma}^{-1} \boldsymbol{Y}
$$

Which is related to the channel model as:

$$
\begin{gathered}
\boldsymbol{\Gamma}=\boldsymbol{M} \boldsymbol{M}^{H}+\boldsymbol{L L}^{H}, \boldsymbol{Y}=\boldsymbol{M} \boldsymbol{L}^{T}+\boldsymbol{L} \boldsymbol{M}^{\boldsymbol{T}} \\
L_{k m}=\delta_{k m}+\varepsilon \sum_{n} X_{k+n} X_{m+n}^{*} K_{n, m-k}, M_{k m}=\varepsilon \sum_{n} X_{k+n} X_{m-n} K_{n, m-k-m}
\end{gathered}
$$

and are described via the following signal-noise coupling matrix: $K_{m n}=\int \mathrm{dz} \sqrt{\Psi_{n}(z) \Psi_{s}(z)} \tilde{\mathrm{C}}_{\mathrm{mn}}$. The received conditional pdf is the main result of the paper: this is the first result of conditional pdf derived for discrete-time fiber-optic channel with memory accurately defined by nonlinear properties of the channel. It allows to model non-circular behaviour of signal distortions and contains precise information about inter-symbol and signalnoise interference.

\section{CONCLUSIONS}

We proposed a general finite memory multivariate channel model for describing nonlinear inter-symbol interfering effects in fibre optic communication channels. The model predicts an exponential decay of the interference with the inter-symbol distance enabling high accuracy for different modulation formats even in highly nonlinear regime. Moreover, we derived the first analytical expression of the conditional pdf that describes signal-interference in fiber-optic channels. Our approach can be used as an information-theoretic tool for estimating the capacity limits of fibre channels, as well as, for the design of efficient compensation algorithms and coding schemes tailored by the nonlinearity.

\section{ACKNOWLEDGEMENTS}

This work has been supported by the EPSRC project UNLOC EP/J017582/1.

\section{REFERENCES}

[1] C. E. Shannon, "A mathematical theory of communication," Bell Syst. Tech. J. 27, 379-423, 623-656 (1948).

[2] A. Splett, C. Kurtzke, and K. Petermann, "Ultimate transmission capacity of amplified optical fiber communication systems taking into account fiber nonlinearities," in Tech. Digest of European Conference on Optical Communication, 1993, paper MoC2.4.

[3] R.-J. Essiambre, G. Foschini, G. Kramer, and P. Winzer, "Capacity limits of information transport in fiberoptic networks," Phys. Rev. Lett. 101, 163901 (2008).

[4] D. J. Richardson, "Filling the light pipe," Science 330, 327-328 (2010).

[5] P. Poggiolini, A. Carena, V. Curri, G. Bosco, and F. Forghieri, "Analytical modeling of non-linear propagation in uncompensated optical transmission links," IEEE Photon. Technol. Lett. 23, 742-744 (2011).

[6] E. Agrell, A. Alvarado, G. Durisi, and M. Karlsson, "Capacity of a nonlinear optical channel with finite memory," J. Lightwave Technol. 16, 2862-2876 (2014).

[7] M. Sorokina, A. Ellis, S.K. Turitsyn, "Optical information capacity processing," chapter in "All-Optical Signal Processing", 325-354 (2015).

[8] E. Agrell, G. Durisi, P. Johannisson, P. "Information-theory-friendly models for fiber-optic channels: A primer". IEEE Information Theory Workshop (ITW), Jerusalem, Israel (2015).

[9] E. Temprana, E. Myslivets, B.P.-P. Kuo, L. Liu, V. Ataie, N. Alic, S. Radic, "Overcoming Kerr-induced capacity limit in optical fiber transmission," Science, Vol. 348 no. 6242 pp. 1445-1448, (2015),

[10] Z. Tao, L. Dou, W. Yan, L. Li, T. Hoshida, J. C. Rasmussen, ”Multiplier-free intrachannel nonlinearity compensating algorithm operating at symbol rate," J. Lightwave Technol., 29, 2570-2581 (2011).

[11] Z. Li, W.-R. Peng, F. Zhu, and Y. Bai, "Optimum quantization of perturbation coefficients for perturbative fiber nonlinearity mitigation," ECOC 2014, We.1.3.4.

[12] A. Ghazisaeidi and R.-J. Essiambre, "Calculation of coefficients of perturbative nonlinear precompensation for Nyquist pulses," ECOC 2014, We.1.3.3. 